\title{
TENDENCIAS EN LA TECNOLOGÍA EDUCATIVA DE NUESTROS DÍAS
}

\author{
TRENDS IN TODAY'S EDUCATIONAL TECHNOLOGY
}

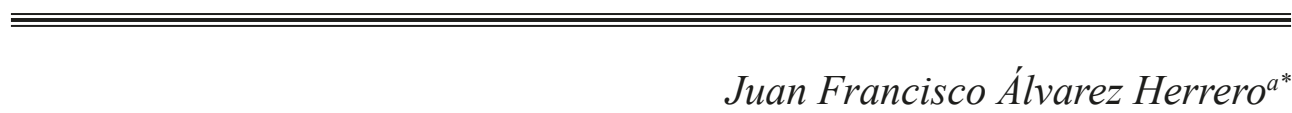

Fechas de recepción y aceptación: 18 de octubre de 2019, 6 de diciembre de 2019

\begin{abstract}
Resumen: La tecnología educativa (TE) en estos últimos años sufre continuos cambios en su campo de acción e investigación. A veces se deja guiar por las modas, por los nuevos avances y recursos que ofrecen las tecnologías de la información y la comunicación (TIC), o bien por aquellas metodologías que adquieren un mayor protagonismo en las aulas. Esto propicia que el campo de estudio e investigación viva sujeto a estas tendencias, lo que se puede observar muy fácilmente si atendemos varios frentes que se hacen eco de esto: por un lado, hacia dónde se dirigen las publicaciones sobre TE (revistas, libros, etc.); por otro, en las temáticas de los trabajos de fin de grado y fin de máster del alumnado de Magisterio y de posgrado en TE. Con el fin de conocer hacia dónde se dirige el interés y la investigación de TE en la actualidad, se analizaron las temáticas y las palabras clave de 95 trabajos fin de máster (TFM) del alumnado de los cursos 17/18 y 18/19 pertenecientes a un máster en Innovación en Tecnología Educativa. Se constata que aquellas áreas, recursos y metodologías más de moda o que están más presentes en nuestros días en las aulas de todas las etapas educativas no universitarias (gamificación, robótica, flipped classroom, aprendizaje basado en juegos, escape room, etc.) copan el mayor interés y suscitan y propician que se continúe investigando sobre ellas entre el alumnado de dicho máster.
\end{abstract}

${ }^{a}$ Facultad de Educación. Universidad de Alicante.

${ }^{*}$ Correspondencia: Universidad de Alicante. Facultad de Educación. Calle del Aeroplano s/n 03690 (San Vicente del Raspeig), Alicante. España.

E-mail: juanfran.alvarez@ua.es 
Palabras clave: tecnología educativa, TIC, investigación en tecnología educativa, investigación en TIC, tecnologías emergentes, metodologías emergentes.

Abstract: Educational technology (ET) has been undergoing continuous changes in the action and research field in the last few years. Sometimes these educational technologies are informed by trends, new information and communication technologies (ICT) advancements and resources, others, by remarking methodologies as used in the classroom. This makes this field of study dependent on these new trends, which can be easily observed in the line taken by research works in journals, books and so, on the one hand, and in the main focus of final undergraduate and postgraduate Teacher Training projects, on the other. With the aim of learning where interest and research in ET is leading to, the subjects and keywords of 95 final projects are thus analyzed, focusing on 17/18 and 18/19 academic years of the master's degree in Innovation and Educational Technology. It is proved that the trendiest areas, resources and methodologies or those which are more frequently present in today's school and high school levels (such as gamification, robotics, flipped classroom, play-based learning, escape rooms, etc.) receive and arouse the greatest interest, and so make it easier for training teachers to continue their research.

Keywords: educational technology, ICT, research in educational technology, research in ICT, emerging technologies, emerging methodologies.

\section{INTRODUCCIÓN}

La tecnología educativa (TE) viene definida como el estudio y la práctica ética de facilitar el aprendizaje y mejorar el rendimiento mediante la creación, el uso y la gestión de procesos y recursos tecnológicos adecuados (Januszewski y Molenda, 2013). El principio fundamental de todo docente pasa por facilitar el aprendizaje y velar por su mejora en su alumnado, y si esto se puede lograr con el uso de la TE, es obligación del profesorado hacer un uso adecuado de ella (Torres y Cobo, 2017). Como su definición describe, comporta el uso y gestión de procesos y recursos tecnológicos. Procesos y recursos que se encuentran en continuo cambio, pues las novedades, mejoras y evolución de estos se producen a un ritmo tan vertiginoso que exige al profesorado estar en una formación y aprendizaje continuo y constante (Grande, Cañón y Cantón, 2016). El uso de las tecnologías de la información y la comunicación (TIC) no solo exige estar en una formación continua y en hacer un buen uso de ellas; también implica estar al corriente de las metodologías y pedago- 
gías que se van introduciendo en las aulas, bien debido a las exigencias de la sociedad y su continuo cambio, bien a causa de la Administración, de la comunidad educativa o de modas que consiguen imponerse muchas veces por encima de argumentos didácticos y pedagógicos.

A tenor de lo aquí hasta ahora expuesto, estamos hablando de una realidad muy compleja, en continuo cambio y que se antoja muy variable, como también lo es la sociedad en la que vivimos, a la que Bauman (2003) llamó modernidad líquida, una sociedad compleja e imprevisible.

Si hacemos un poco de historia en la trayectoria de la TE, veremos que se ha pasado de momentos donde se entendía como una novedad que el simple hecho de disponer de un recurso tecnológico significara estar ya cumpliendo con esa misión de facilitar y mejorar el aprendizaje. Después, cuando ya no era tan novedoso tener dispositivos y recursos, se pasó por una época donde se requería de una formación en su uso y manejo. Ello propició que proliferasen formaciones centradas en el uso de determinadas herramientas y recursos (Llorente, 2008), en las que muchas veces se daba preferencia a esto por delante de otras cuestiones consideradas secundarias, como determinar si el uso de estos recursos en sus momentos y circunstancias estaba justificado o, lo que es lo mismo, si producía una mejora en el aprendizaje o facilitaba la comprensión de lo enseñado (Cabero, 2016). La formación en TIC pasó, como decíamos, a estar centrada en conocer el uso de suites ofimáticas o de determinado software sin plantearse qué uso pedagógico se podía hacer de él. Recientemente, tanto la administración educativa como los propios centros educativos se han dado cuenta de la importancia de anteponer en la formación en TIC su valor pedagógico antes que considerar única y exclusivamente el manejo y uso de la herramienta o recurso en sí (Cabero, 2017). Se trata de formar a los docentes en saber hacer un uso de la tecnología que permita integrarla en los procesos de enseñanza-aprendizaje, permitiendo una mejora en estos (Tondeur, van Braak, Siddiq y Scherer, 2016).

Una evolución de desarrollo similar a la de la TE se observa cuando se analizan las tendencias de estudio e investigación llevadas a cabo sobre la educación a distancia (Zawacki-Richter y Naidu, 2016), ya que, en definitiva, educación y tecnología van ligadas, sea ya de forma presencial o a distancia, y han estado sometidas a los mismos cambios e innovaciones. 
Pero, en la actualidad, se ha dado un paso más allá, y parece que la preocupación y el interés en la TE se han visto desplazados por una mayor focalización en las metodologías presentes en todo proceso de enseñanza-aprendizaje (Gros y Noguera, 2015). Unas metodologías que buscan que sean activas, donde se confiera un mayor protagonismo al alumnado, quien debe construir su propio aprendizaje, donde el profesorado adquiere un rol de guía, de orientador y favorecedor de este proceso. Con ello, la TE ha quedado, podríamos decir, relegada al papel secundario de hacer un uso de ella solo al servicio de estas metodologías, demostrándose, eso sí, que si se hace un buen uso de la tecnología, se consigue una mejora considerable en el aprendizaje del alumnado (Mattar y Ramos, 2019). Y, por otro lado, si su uso no es necesario o no está justificado, se prescinde de la TE. Por explicarlo de una manera muy sencilla, en los últimos años hemos pasado de mucha tecnología y poca metodología a mucha metodología y poca tecnología. Pero todo ello lo que está propiciando son nuevas formas de entender la educación, donde ya se habla de que otra educación es posible, de una revolución de la enseñanza y el aprendizaje.

Cierto es que estas consideraciones que estamos haciendo no representan a la totalidad del colectivo o comunidad educativa, y que siempre habrá casos aislados donde la tecnología sigue siendo la protagonista, sin contemplar su uso metodológico. Pero, como decíamos, principalmente las modas han venido a traernos al mundo de la educación las metodologías activas, y es ahora a la tecnología a la que le toca estar al servicio de estas.

Para tomarle el pulso a la TE en nuestros días, o bien, podemos buscar o realizar análisis sobre las investigaciones y estudios en los últimos años como indicadores de en qué ámbitos y hacia dónde está yendo la TE (Baydas, Kucuk, Yilmaz, Aydemir y Goktas, 2015; García-Peñalvo, 2015; Marín, Zawacki-Richter, Pérez y Salinas, 2017). Al investigador se le hace necesario comunicar, poner en abierto el conocimiento y así permitir el intercambio y la construcción de nuevo conocimiento (Salinas, 2019). O bien, otra de las opciones que podemos contemplar pasa por analizar otro tipo de indicadores, como pueden ser las redes sociales (Roig y Álvarez, 2019), o más directamente analizar cuáles son las temáticas de las investigaciones que a modo de trabajo final de máster realiza el alumnado de másteres en Innovación y Tecnología Educativa. 
Si bien las temáticas se pueden ver influidas a la hora de su elección por las asignaturas impartidas en el máster y su profesorado, siempre hay un porcentaje mayor de elección que obedece a aquellos aspectos en los que surge el interés de la persona, del área y/o etapa en la que sea docente o de aquellos temas en los que bien por imposición del centro, bien por las modas del momento generan el interés del alumnado del máster.

Dado que el autor de esta investigación es profesor en varias universidades y en una de ellas lo es de una asignatura de un máster en Innovación en Tecnología Educativa, ha tenido acceso durante los dos últimos cursos académicos a los TFM del alumnado. De estos TFM y de los datos que de ellos y sus autores se desprenden, podemos hacernos una idea bastante objetiva de los temas de interés que mueven la TE de nuestros días. Si bien desde el máster se ofrece al alumnado orientación hacia posibles temas que elegir, es el alumnado quien finalmente de manera libre y sin presiones escoge aquel tema que desea trabajar. Y esta elección o deseo no es difícil ver que se hace en función de los propios intereses del alumnado, y por tanto responde bien a la etapa en la que se encuentran trabajando, a las metodologías con las que están familiarizados o con las que van a trabajar, o bien de aquellos intereses, recursos, o metodologías que están de moda y van a tener una próxima implantación en su labor docente o que van a querer implantar cuando estén en activo. Pues no podemos olvidar que el perfil del alumnado de este máster corresponde bien a profesorado en activo de todas las etapas educativas, bien a futuros docentes en búsqueda de trabajo pero con una especialidad y unos intereses en el uso de las TIC bien marcado.

Se trata, pues, de un excelente indicador que nos va a permitir conocer cuáles son los ámbitos de interés e investigación de la TE de nuestros días.

\section{Objetivos}

En esta investigación nos planteamos como objetivo principal conocer cuáles son los ámbitos de estudio e investigación que dentro de la TE están marcando el interés en estos últimos años. Para ello vamos a analizar los TFM del alumnado de los dos últimos cursos académicos de un máster en 
Innovación Tecnológica en Educación, pues esto nos permitirá conocer el fin o la meta que nos hemos marcado. A su vez, se pretende:

- Establecer algún tipo de relación entre las temáticas de mayor interés y la etapa educativa o el género del colectivo que las elige.

- Comprobar si existe alguna relación entre los temas o ámbitos de mayor interés y las modas pedagógicas que actualmente encontramos en las aulas.

\section{Metodología}

La muestra analizada consta de 95 TFM correspondientes a un total de 71 mujeres y 24 hombres. Estamos hablando del alumnado del máster en Innovación Tecnológica en Educación de la Universidad Católica de Valencia en sus promociones $17 / 18$ y 18/19 y que presentaron y defendieron sus TFM en primera o segunda convocatoria.

Se procedió a realizar un análisis cualitativo descriptivo de los títulos de dichos trabajos, las palabras clave, el género de su autor/a, la etapa educativa en la que se centra el trabajo y su área temática. Para recoger los datos y analizar las variables cuantitativas se utilizó el programa SPSS Stadistics v. 25, mientras que para el análisis cualitativo se utilizaron dos aplicaciones web de creación de nubes de palabras: Wordart.com y nubedepalabras.es.

Con SPSS se obtuvieron frecuencias, porcentajes y otros datos estadísticos de interés sobre las variables: sexo, etapa educativa del tema, temática, etc. Por el contrario, con las aplicaciones de nubes de palabras se analizó el texto resultante de unir todos los títulos de los 95 TFM analizados, así como el texto producto de unir todas las palabras clave de todos los trabajos. Estas aplicaciones permiten el recuento de palabras y la clasificación en función de su repetición o frecuencia, así como mostrar de forma gráfica estos mismos resultados a modo de nube de palabras, donde aquellas que más veces se repiten aparecen con un tamaño de letra mayor y así gradualmente hasta las que aparecen en menor número, que tienen el tamaño de letra más pequeño. 


\section{RESUlTADOS Y DISCUSIÓN}

\subsection{Resultados cuantitativos}

Tal y como ya se ha comentado, se dispuso de 95 TFM entre las dos últimas ediciones del máster, 40 en la de 17/18 y 55 en la del 18/19. En la tabla 1 se puede observar su distribución y porcentajes por ediciones, convocatorias y sexo.

En dicha tabla 1 se puede observar cómo se cumple un porcentaje medio que oscila entre el 63 y el $84 \%$ para las mujeres, y entre el 33 y el $16 \%$ para los hombres, dependiendo de las ediciones y convocatorias. No obstante, si vemos el porcentaje medio que se obtiene del conjunto de convocatorias y ediciones, se obtiene el 74,7 \% para las mujeres y el 25,3\% para los hombres, lo que se corresponde en gran medida con el $72,1 \%$ para mujeres y el $27,9 \%$ para hombres que señalan las estadísticas del Ministerio de Educación y Formación Profesional con fecha de octubre de 2019 sobre el porcentaje y distribución del profesorado por sexo y tipo de enseñanza del curso 2017-2018 y, más concretamente, para el profesorado en enseñanzas de régimen general no universitarias.

Con estos datos podemos afirmar que la muestra que analizamos refleja la situación real que vive nuestro sistema educativo y que ya se viene observando desde hace años, y que nos ofrece un porcentaje medio.

TABLA 1

Distribución por sexo del alumnado según el curso y convocatoria

\begin{tabular}{|c|c|c|c|c|c|c|c|c|c|c|c|c|c|c|}
\hline & \multicolumn{6}{|c|}{ Edición 17/18 } & \multicolumn{6}{|c|}{ Edición 18/19 } & \multicolumn{2}{|c|}{ TOTAL } \\
\hline & 1. ${ }^{a} \mathrm{C}$ & $\%$ & $2 .{ }^{a} \mathrm{C}$ & $\%$ & $T$ & $\%$ & 1. ${ }^{a} \mathrm{C}$ & $\%$ & $2 .{ }^{a} \mathrm{C}$ & $\%$ & $T$ & $\%$ & $T$ & $\%$ \\
\hline 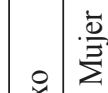 & 14 & 63,6 & 12 & 66,7 & 26 & 65 & 24 & 80 & 21 & 84 & 45 & 81,8 & 71 & 74,7 \\
\hline 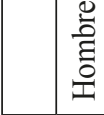 & 8 & 36,4 & 6 & 33,3 & 14 & 35 & 6 & 20 & 4 & 16 & 10 & 18,2 & 24 & 25,3 \\
\hline Total & 22 & 100,0 & 18 & 100,0 & 40 & 100 & 30 & 100 & 25 & 100 & 55 & 100,0 & 95 & 100,0 \\
\hline
\end{tabular}


Por otro lado, también se analizó el ámbito de estudio de los TFM, es decir, la etapa o conjunto de ellas en las que se centraba cada uno de los trabajos, cuyos resultados se pueden ver en la tabla 2 .

TABLA 2

Distribución por ámbito de estudio en los TFM

\begin{tabular}{|l|c|c|}
\hline & $N$ & $\%$ \\
\hline Educación Infantil & 21 & 22,1 \\
\hline Educación Primaria & 37 & 38,9 \\
\hline Educación Secundaria + Bachillerato & 22 & 23,2 \\
\hline Ciclos de Formación Profesional & 3 & 3,2 \\
\hline Profesorado & 3 & 3,2 \\
\hline Centro escolar (EE. no universitarias) & 9 & 9,5 \\
\hline TOTAL & 95 & 100,0 \\
\hline
\end{tabular}

De esta tabla 2 se desprende que todos los ámbitos están representados y siguen una proporcionalidad muy semejante a la que existe en nuestro sistema educativo, tal y como se desprende nuevamente de los datos estadísticos del Ministerio de Educación y Formación Profesional.

Dentro del estudio cuantitativo que se realizó, también se consideró interesante categorizar las diferentes áreas temáticas que eran objeto de investigación en cada uno de los TFM analizados, pudiéndose dar 2 o incluso 3 áreas temáticas a la vez, cuyos resultados se muestran en la tabla 3.

TABLA 3

Distribución y porcentajes por áreas o temáticas de estudio en los TFM

\begin{tabular}{|l|c|c|}
\hline \multicolumn{1}{|c|}{ Temática o área principal de estudio } & $N$ & $\%$ \\
\hline TIC & 32 & 33,7 \\
\hline Gamificación + ABJ & 16 & 16,8 \\
\hline Robótica & 12 & 12,6 \\
\hline Realidad aumentada & 8 & 8,4 \\
\hline
\end{tabular}




\begin{tabular}{|l|l|l|}
\hline \multicolumn{1}{|c|}{ Temática o área principal de estudio } & $N$ & $\%$ \\
\hline Youtube+video+flipped classroom & 6 & 6,3 \\
\hline Moodle & 5 & 5,3 \\
\hline Móviles - mlearning & 5 & 5,3 \\
\hline Podcast (audio, radio, sonido) & 5 & 5,3 \\
\hline PLE & 3 & 3,2 \\
\hline Escape classroom +breakout edu & 3 & 3,2 \\
\hline Competencia digital & 3 & 3,2 \\
\hline Inclusión & 3 & 3,2 \\
\hline TEA + Asperger & 3 & 3,2 \\
\hline Seguridad en internet & 2 & 2,1 \\
\hline Aprendizaje cooperativo & 2 & 2,1 \\
\hline Impresión 3D & 2 & 2,1 \\
\hline Equipamiento informático & 1 & 1,1 \\
\hline Movimiento maker & 1 & 1,1 \\
\hline Marketing educativo & 1 & 1,1 \\
\hline Alumnado disruptivo & 1 & 1,1 \\
\hline Webquest & & 1,1 \\
\hline Coaching educativo & 1,1 \\
\hline
\end{tabular}

Como era previsible, tratándose de un máster en Innovación en Tecnología Educativa, un gran número de trabajos realizan sus investigaciones en la temática general de las TIC, sobre cómo introducirlas o implementarlas en un centro, una etapa o una clase en concreto. Y después podemos observar cómo hay tres temas que destacan por detrás y que están muy de moda en la actualidad: la gamificación y el aprendizaje basado en el juego (ABJ), la robótica y la realidad aumentada. Tal y como introducíamos en este estudio, son muchos los docentes que, inquietos y con ganas de innovar, introducen otras metodologías más activas a su forma de dar clase, entre ellas, ligadas con mayor o menor suerte, las TIC. 
Sirva este primer análisis de temáticas para comprobar lo que más adelante ya profundizaremos, que es la presencia cada vez mayor de las metodologías por delante de la tecnología. Hecho este que no criticamos, sino que, al contrario, demuestra cómo la tecnología puede ayudar a enriquecer y mejorar el aprendizaje del alumnado, incluso mediante el uso de metodologías emergentes o más activas.

\subsection{Resultados cualitativos}

Con los títulos y las palabras clave de estos 95 trabajos, procedimos, como ya hemos dicho, a crear dos textos, uno con todos los títulos y otro con todas las palabras clave. En estas últimas, sustituimos los espacios que pudiese haber entre palabras dentro de una misma palabra clave por guiones bajos para permitir que la aplicación de nubes de palabras las pudiese considerar como una única palabra, en aquellas consistentes en dos o más palabras.

De los títulos y palabras con significado concreto, después de eliminar conectores, artículos, preposiciones, adverbios, etc., los 20 términos (no se consideran los restantes por no extender más esta investigación y por carecer de interés) con mayor repetición o frecuencia se pueden consultar en la tabla 4.

TABLA 4

20 palabras con mayor índice de repetición en los títulos de los TFM

\begin{tabular}{|l|c|}
\hline \multicolumn{1}{|c|}{ PALABRA } & $N$ \\
\hline Educación & 35 \\
\hline TIC & 27 \\
\hline Aprendizaje & 25 \\
\hline Primaria & 20 \\
\hline Infantil & 19 \\
\hline Propuesta & 19 \\
\hline Aula & 18 \\
\hline Herramienta & 12 \\
\hline Gamificación & 12 \\
\hline
\end{tabular}




\begin{tabular}{|l|c|}
\hline \multicolumn{1}{|c|}{ PALABRA } & $N$ \\
\hline Alumnado & 10 \\
\hline Didáctica & 9 \\
\hline Enseñanza & 9 \\
\hline Realidad & 8 \\
\hline Aumentada & 8 \\
\hline Tecnología & 8 \\
\hline Recurso & 8 \\
\hline Educativa & 8 \\
\hline Robótica & 8 \\
\hline Metodología & 7 \\
\hline Trabajar & 7 \\
\hline
\end{tabular}

De igual modo, pero de una forma más gráfica, podemos observar en la figura 1 el resultado de la repetición de estas palabras en los títulos de los TFM, apareciendo con mayor tamaño aquellas con mayor índice de repetición y menguando este conforme se van repitiendo menos.

\section{FIGURA 1}

Nube de palabras resultante de analizar los titulos de los TFM

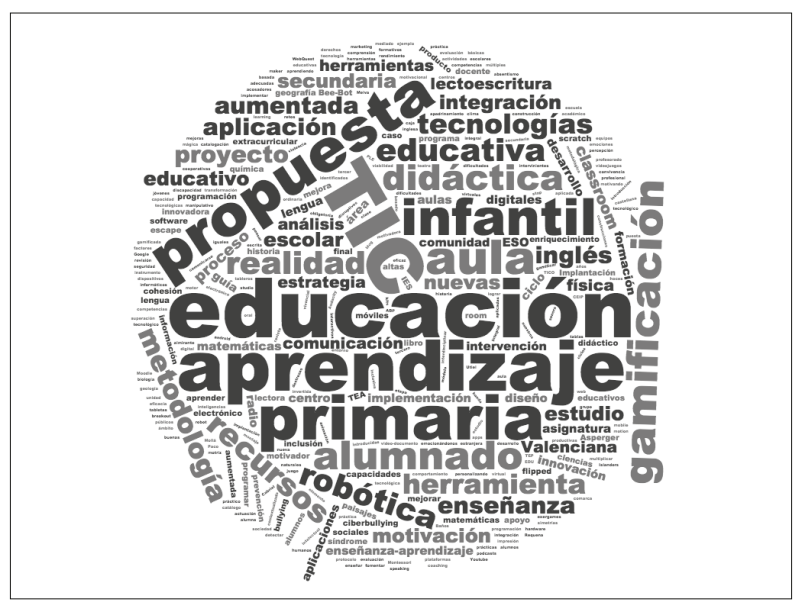


Se hizo lo mismo con las palabras clave de los 95 trabajos analizados, teniendo la precaución ya comentada de poner guiones bajos en vez de espacios para que la aplicación de nubes de palabras utilizada pudiese considerar aquellas palabras clave formadas por 2 o más palabras como una sola. El resultado, primero del recuento de dichas palabras y de forma gráfica después, se puede ver en la tabla 5 y la figura 2 respectivamente.

TABLA 5

20 palabras con mayor índice de repetición en las palabras clave de los TFM

\begin{tabular}{|l|l|}
\hline \multicolumn{1}{|c|}{ PALABRA } & $N$ \\
\hline TIC & 46 \\
\hline Educación_Primaria & 21 \\
\hline Educación_Infantil & 20 \\
\hline ESO & 19 \\
\hline Gamificación & 17 \\
\hline Educación & 16 \\
\hline Motivación & 12 \\
\hline Robótica & 11 \\
\hline Trabajo_cooperativo & 9 \\
\hline Realidad_aumentada & 8 \\
\hline Innovación & 8 \\
\hline Nuevas_Tecnologías & 7 \\
\hline Competencia_Digital & 5 \\
\hline Flipped_classroom & 5 \\
\hline Aprendizaje_basado_en_juegos & 4 \\
\hline Enseñanza-aprendizaje & 4 \\
\hline Lectoescritura & 4 \\
\hline Matemáticas & 4 \\
\hline Alumnado & 4 \\
\hline ABP & 4 \\
\hline
\end{tabular}


Observamos nuevamente palabras tales como TIC, educación, Educación Primaria, Educación Infantil, gamificación, robótica o alumnado, que están también entre las que más se repiten en las palabras clave de los TFM. Si bien cabe destacar que hay palabras que no aparecen en los títulos y que sí lo hacen en las palabras clave, concretamente: motivación, ESO, innovación, trabajo cooperativo; incluso palabras que hacen referencia a áreas o temáticas muy concretas como son matemáticas y lectoescritura.

FIGURA 2

Nube de palabras resultante de analizar las palabras clave de los TFM

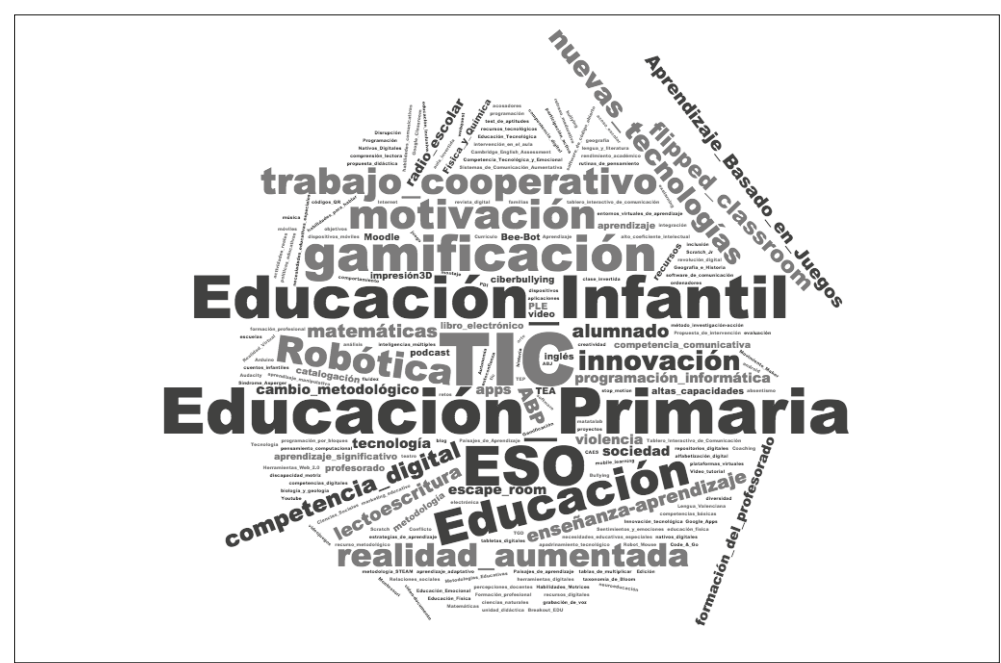

Por último, quisimos cruzar los datos de al menos cuatro de las metodologías que más se repiten: gamificación $+\mathrm{ABJ}$, realidad aumentada, robótica y flipped classroom, con las etapas educativas en las que se habían investigado y con el sexo del/a investigador/a. Los resultados se pueden ver en las tablas 6 y 7 . En la tabla 6, se puede observar cómo la robótica y la realidad aumentada tienen un mayor éxito en la Educación Infantil, mientras que la flipped classroom y la gamificación+ABJ triunfan en Educación Primaria. 
TABLA 6

Correlación entre cuatro metodologías y las etapas educativas en las que se estudian

\begin{tabular}{|l|c|c|c|c|c|c|c|}
\hline & Infantil & Primaria & Secund. & FP & Profes. & Centro & TOTAL \\
\hline $\begin{array}{l}\text { Gamificación } \\
+ \text { ABJ }\end{array}$ & $\begin{array}{c}4 \\
(25 \%)\end{array}$ & $\begin{array}{c}7 \\
(43,8 \%)\end{array}$ & $\begin{array}{c}5 \\
(31,2 \%)\end{array}$ & $\begin{array}{c}0 \\
(0 \%)\end{array}$ & $\begin{array}{c}0 \\
(0 \%)\end{array}$ & $\begin{array}{c}0 \\
(0 \%)\end{array}$ & $\begin{array}{c}16 \\
(100 \%)\end{array}$ \\
\hline Robótica & 9 & 2 & 1 & 0 & 0 & 0 & 12 \\
$(75 \%)$ & $(16,7 \%)$ & $(8,3 \%)$ & $(0 \%)$ & $(0 \%)$ & $(0 \%)$ & $(100 \%)$ \\
\hline RA & 4 & 2 & 0 & 1 & 0 & 1 & \\
\hline $\begin{array}{l}\text { Flipped class- } \\
\text { room }\end{array}$ & 0 & 4 & 1 & 0 & 1 & 0 & 0 \\
$(50 \%)$ & $(25 \%)$ & $(0 \%)$ & $(12,5 \%)$ & $(0 \%)$ & $(12,5 \%)$ & $8(100 \%)$ \\
\hline
\end{tabular}

TABLA 7

Correlación entre cuatro metodologías y el sexo del/a investigador/a

\begin{tabular}{|l|c|c|c|c|c|}
\hline & \multicolumn{2}{|c|}{ HOMBRE } & \multicolumn{2}{c|}{ MUJER } & TOTAL \\
\hline & $N$ & $\%$ & $N$ & $\%$ & \\
\hline Gamificación+ABJ & 11 & 68,8 & 5 & 31,3 & 16 \\
\hline Robótica & 1 & 8,3 & 11 & 91,7 & 12 \\
\hline Realidad aumentada & 1 & 12,5 & 7 & 87,5 & 8 \\
\hline Flipped classroom & 2 & 33,3 & 4 & 66,7 & 6 \\
\hline
\end{tabular}

Respecto al género/sexo del/a investigador/a, se comprueba que las mujeres son mayoritariamente las que investigan sobre robótica y realidad aumentada, mientras que en las otras dos metodologías los porcentajes coinciden con el porcentaje de presencia de docentes de uno y otro sexo. Si bien ya hemos comentado previamente que la robótica y la realidad aumentada se daban sobre todo en la etapa de Educación Infantil, y precisamente en esta etapa es donde mayor porcentaje de docentes mujeres hay respecto a los docentes hombres, es cierto que unos porcentajes tan elevados, del 91,7 y del 87,5\%, nos permiten afirmar que estas dos metodologías tienen una mayor incidencia en la investigación e interés de las docentes que de los docentes. 


\section{Conclusiones}

Ante un máster en Innovación Tecnológica en Educación, era previsible esperar que la gran mayoría de los TFM de su alumnado tuviesen como temática de investigación el uso o la implementación de las TIC, bien en el aula, bien en una etapa o en todo un centro. Lo que ya no era tan previsible, y hemos demostrado con esta pequeña investigación del análisis de las temáticas de 95 TFM, es cómo la tecnología educativa se está acercando cada vez más a las llamadas metodologías emergentes o activas, y cómo se busca de qué manera las TIC pueden ayudar o generar un valor añadido al aprendizaje del alumnado con la implementación de estas metodologías.

Sí queda patente y demostrado que la importancia de la educación y más concretamente del aprendizaje y del uso de las TIC están presentes en las investigaciones del alumnado del máster. Pues tanto de los resultados cualitativos de los títulos de sus investigaciones como de las palaras clave de dichos trabajos se constata que aprendizaje, educación, TIC, tecnología e innovación son las palabras que más se repiten. Pero también adquieren un gran protagonismo las metodologías de las que venimos hablando.

Estas metodologías, como ha quedado ampliamente argumentado en el análisis de los resultados obtenidos, son básicamente: la gamificación + aprendizaje basado en el juego, la robótica, la realidad aumentada, el flipped classroom y, en menor medida, el aprendizaje colaborativo, el aprendizaje basado en proyectos, los podcasts, etc. Por otro lado, sorprende que no hayan adquirido una mayor relevancia y mejores resultados metodologías y recursos que también están bien posicionados en la actualidad educativa, como son la impresión 3D, el movimiento maker o la seguridad en internet. Precisamente, la seguridad en internet y los casos de ciberbullying o de acoso digital son muy preocupantes y requerirían un mayor interés, máxime cuando es en estos temas donde mayor carencia de formación e investigación hay en la actualidad.

El objetivo principal que nos marcábamos sobre conocer los ámbitos de estudio actuales de la tecnología educativa en estos últimos años queda, pues, recogido en estas conclusiones. Las TIC en general siguen siendo el tema estrella de investigación, si bien se observan que crece la tendencia hacia el estudio de la repercusión e implementación de las TIC en metodologías activas como la gamificación, la robótica, la realidad aumentada, etc., pasando 
las temáticas que en otras épocas estaban más de moda, como el Moodle, el PLE (Entorno Personal de Aprendizaje), el coaching educativo o incluso la competencia digital del alumnado y profesorado, a ocupar posiciones más discretas y alejadas de los principales temas de interés.

En los estudios más recientes sobre las tendencias en tecnología educativa (Baydas et al., 2015), resultaban como los más investigados los enfoques o teorías de aprendizaje (podemos considerarlos como las metodologías de las que estamos hablando) y los entornos de aprendizaje (que como ya hemos visto en nuestra investigación, están quedando relegados en la actualidad a un segundo plano). Hay que tener en cuenta que los casi cinco años que han pasado desde el estudio de Baydas et al. (2015) hasta el nuestro hay toda un eternidad cuando hablamos de TE por el continuo cambio al que esta está sometido.

Tanto para la etapa educativa como para el género del profesorado investigador, se observa que la robótica y la realidad aumentada tienen un marcado carácter femenino y son más frecuentes en la etapa de Educación Infantil. Si bien, como es sabido, la etapa de Educación Infantil está más ampliamente copada por maestras que por maestros, que son una escasa minoría, los porcentajes obtenidos nos permiten afirmar que estas dos metodologías generan mayor interés entre las mujeres que entre los hombres. De las otras dos metodologías, la gamificación y el flipped classroom, podríamos decir que siguen dentro de la proporcionalidad que tanto de etapa educativa como de sexo de los/las investigadores/as hay en nuestro sistema educativo.

Por último, debemos confirmar lo que ya presumíamos en la introducción de este estudio. La tecnología educativa de nuestros días está dando paso, poco a poco pero con paso firme, al estudio del uso de las TIC en las metodologías "de moda" o metodologías activas, suscitando un interés creciente en ellas y relegando a temas que antaño tenían un mayor interés.

En conclusión, corren nuevos tiempos para la TE y los nuevos temas van relegando a otros ya más en desuso. Algo que es totalmente previsible y entendible, pues estamos hablando de un área, la TE, en continuo cambio, en continua evolución, y que tiene que adaptarse a los tiempos, a los avances tanto tecnológicos como pedagógicos que la sociedad en general y la comunidad educativa en particular le exigen.

Pero lejos de obedecer a modas o tendencias, la escuela de nuestros días debe procurar que su objetivo prioritario sea favorecer y mejorar el aprendi- 
zaje de su alumnado para formar personas, ciudadanos del mañana. Y para conseguirlo debe acudir a aquellos recursos, a aquellas metodologías y estrategias que mejor se adapten en cada momento y a su contexto particular, siendo cada escuela, cada caso, un mundo muy particular que no obedece ni a franquicias ni a estereotipos marcados o dictados por cualquier agente externo al de la propia comunidad educativa.

Asimismo, somos conscientes de las limitaciones de este estudio, no solo en cuanto a la muestra recogida, sino también, y como ya hemos advertido anteriormente, porque la TE cambia constantemente y lo hace a un ritmo tan vertiginoso que metodologías y recursos que están de actualidad cuando se ha realizado esta investigación pueden haber pasado a estar desfasadas o hayan desaparecido, cuando años o incluso meses después se estén leyendo estas conclusiones; o bien puede haber aparecido nuevas metodologías, recursos o modas que ya existían pero que habían quedado en el olvido. Así es el mundo de la educación, el de la tecnología y, más concretamente, el de la tecnología educativa.

Como líneas de investigación futuras, nos planteamos revisar de nuevo, en un plazo corto de tiempo, cuáles son los temas de interés de estas investigaciones en un futuro, si coinciden o aparecen nuevos, así como ampliar la muestra de estudio para obtener unos resultados más representativos.

\section{BIBLIOGRAFÍA}

Bauman, Z. (2003). Modernidad líquida. México: Fondo de Cultura Económica.

Baydas, O., Kucuk, S., Yilmaz, R. M., Aydemir, M. y Goktas, Y. (2015). Educational technology research trends from 2002 to 2014. Scientometrics 105(1), 709-725. doi: 10.1007/s11192-015-1693-4.

CABEro, J. (2016). ¿Qué debemos aprender de las pasadas investigaciones en Tecnología Educativa? RIITE. Revista Interuniversitaria de Investigación en Tecnología Educativa 0, 23-33. doi: 10.6018/riite/2016/256741.

CABero, J. (2017). La formación en la era digital: ambientes enriquecidos por la tecnología. Gestión de la Innovación en Educación Superior II(2), 41-64. 
García-Peñalvo, F. J. (2015). Mapa de tendencias en Innovación Educativa. Education in the knowledge society 16(4), 6-23.

Grande, M., Cañón, R. y CANTÓN, I. (2016). Tecnologías de la información y la comunicación: Evolución del concepto y características. IJERI: International Journal of Educational Research and Innovation 6, 218-230.

Gros, B. y Noguera, I. (2015). Mirando el futuro: Evolución de las tendencias tecnopedagógicas en Educación Superior. Campus virtuales 2(2), 130-140.

Llorente Cejudo, M. d. C. (2008). Aspectos fundamentales de la formación del profesorado en TIC. Pixel-Bit 31, 121-130.

Januszewski, A. y Molenda, M. (eds.) (2008). Educational technology: A definition with commentary. Nueva York: Lawrence Erlbaum Associates.

Marín, V., Zawacki-Richter, O., Pérez, A. y Salinas, J. (2017). Tendencias en el ámbito de la tecnología educativa en Iberoamérica: 20 años de la revista edutec-e. EDUTEC, Revista Electrónica de Tecnología Educativa 59.

MatTAR, J. y Ramos, D. K. (2019). Active methodologies and digital technologies. International Journal of Innovation Education and Research 7(3), $1-12$.

Roig-Vila, R. y Álvarez Herrero, J. F. (2019). Repercusión en Twitter de las metodologías activas ABP, Flipped Classroom y Gamificación. RIED. Revista Iberoamericana de Educación a Distancia 22(2), pp. 79-96. doi: 10.5944/ried.22.2.23272.

SALINAS, J. (2019). Investigación en Tecnología Educativa y avance del conocimiento abierto. Revista Interuniversitaria de Investigación en Tecnología Educativa 6, 8-11. doi: 10.6018/riite.386251.

Tondeur, J., van Braak, J., Siddie, F. y Scherer, R. (2016). Time for a new approach to prepare future teachers for educational technology use: Its meaning and measurement. Computers \& Education 94, 134-150. doi: 10.1016/j.compedu.2015.11.009.

Torres, P. C. у Сoвo, J. K. (2017). Tecnología educativa y su papel en el logro de los fines de la educación. Educere 21(68), 31-40.

ZaWACKi-Richter, O. y Naidu, S. (2016). Mapping research trends from 35 years of publications in Distance Education. Distance Education 37(3), 245-269. 